\title{
Telomere alterations in neurofibromatosis type 1-associated solid tumors
}

\author{
Fausto J. Rodriguez 1,2,3* ${ }^{*}$, Mindy K. Graham', Jacqueline A. Brosnan-Cashman', John R. Barber ${ }^{5}$, Christine Davis ${ }^{1}$, \\ M. Adelita Vizcaino ${ }^{1}$, Doreen N. Palsgrove ${ }^{1,3}$, Caterina Giannini ${ }^{6}$, Melike Pekmezci ${ }^{7}$, Sonika Dahiya ${ }^{8}$, Murat Gokden ${ }^{9}$, \\ Michael Noë ${ }^{1}$, Laura D. Wood ${ }^{1,3}$, Christine A. Pratilas ${ }^{3}$, Carol D. Morris ${ }^{10}$, Allan Belzberg ${ }^{11}$, Jaishri Blakeley ${ }^{3,4}$ and \\ Christopher M. Heaphy
}

\begin{abstract}
The presence of Alternative lengthening of telomeres (ALT) and/or ATRX loss, as well as the role of other telomere abnormalities, have not been formally studied across the spectrum of NF1-associated solid tumors. Utilizing a telomerespecific FISH assay, we classified tumors as either ALT-positive or having long (without ALT), short, or normal telomere lengths. A total of 426 tumors from 256 NF1 patients were evaluated, as well as 99 MPNST tumor samples that were sporadic or of unknown NF1 status. In the NF1-glioma dataset, ALT was present in the majority of high-grade gliomas: 14 (of 23; 60\%) in contrast to only 9 (of 47; 19\%) low-grade gliomas ( $p=0.0009$ ). In the subset of ALT-negative glioma cases, telomere lengths were estimated and we observed 17 (57\%) cases with normal, 12 (40\%) cases with abnormally long, and only 1 (3\%) case with short telomeres. In the NF1-associated malignant nerve sheath tumor (NF1-MPNST) set $(n=75)$, ALT was present in $9(12 \%)$. In the subset of ALT-negative NF1-MPNST cases, telomeres were short in 9 (38\%), normal in 14 (58\%) and long in 1 (3\%). In the glioma set, overall survival was significantly decreased for patients with ALT-positive tumors $(p<0.0001)$. In the NF1-MPNST group, overall survival was superior for patients with tumors with short telomeres $(p=0.003)$. ALT occurs in a subset of NF1-associated solid tumors and is usually restricted to malignant subsets. In contrast, alterations in telomere lengths are more prevalent than ALT.
\end{abstract}

Keywords: NF1, ATRX, Alternative lengthening of telomeres, Glioma, MPNST

\section{Introduction}

Patients with neurofibromatosis type 1 (NF1) are prone to develop a variety of neoplasms [4]. The most common central nervous system (CNS) tumors in these patients are pilocytic astrocytomas (PA) which frequently involve the optic pathways. However, other CNS tumor types may also develop [38] (reviewed in Nix et al. [29]). The specific genetic drivers of these tumors are just beginning to be characterized, in contrast to gliomas developing in a sporadic setting, for which more information is known [3].

Telomeres consist of a repetitive hexameric DNA sequence $\left(\mathrm{TTAGGG}_{\mathrm{n}}\right.$ ) bound by the shelterin protein

\footnotetext{
*Correspondence: frodrig4@jhmi.edu

'Departments of Pathology, Johns Hopkins University School of Medicine, Sheikh Zayed Tower, Room M2101, 1800 Orleans Street, Baltimore, MD 21231, USA

${ }^{2}$ Departments of Ophthalmology, Johns Hopkins University School of Medicine, Sheikh Zayed Tower, Room M2101, 1800 Orleans Street, Baltimore, MD 21231, USA

Full list of author information is available at the end of the article
}

complex. Since telomeres progressively shorten with each division cycle, cancer cells require activation of a telomere maintenance mechanism. The predominant mechanism is the upregulation of telomerase, which may be accomplished by numerous genetic means [2, 19]. In contrast, a subset of cancers use the telomerase-independent Alternative Lengthening of Telomeres (ALT) [13] mechanism, which is mediated by homology directed repair. These unique ALT-associated features include the presence of ultra-bright telomeric foci, dramatic cell-to-cell and intracellular telomere heterogeneity, and the presence of single stranded extrachromosomal circles containing the C-rich telomere repeat sequence (C-circles). Even in the absence of ALT, variations in telomere length are being increasingly recognized as a prognostic factor in cancer $[14,41]$.

Prior studies have related alterations in the alpha thalassemia/mental retardation syndrome X-linked (ATRX), death domain-associated protein (DAXX), or SWI/SNF related, matrix associated, actin dependent regulator of 
chromatin (SMARCAL1) genes with ALT in some cancers $[9,12]$. ATRX mutations and ALT are associated with specific molecular subgroups of brain tumors [28]. We reported for the first time a high frequency of ATRX loss and ALT in high-grade and diffuse gliomas developing in NF1 patients [39], and more recent studies have also documented ATRX mutations in aggressive NF1associated gliomas [8, 37]. Genomic studies have identified alterations in SUZ12 and EED in the majority of malignant nerve sheath tumors (MPNST) [21, 43]. These mutations result in profound epigenetic alterations, including loss of H3K27 trimethylation [33].

While most MPNSTs maintain their telomeres presumably through increased telomerase activity [25], recent studies have demonstrated abnormally short telomeres in MPNSTs, in contrast to neurofibromas [18]. Conversely, a global study of telomere lengths across 6835 tumors (31 tumor types) documented that two tumor types in particular, glioma and sarcomas, are characterized by longer telomeres [34]. In the current study, we interrogate the full spectrum of NF1-associated solid tumors for telomere alterations and possible associations with clinicopathologic characteristics and outcomes.

\section{Materials and methods}

\section{Patients and tumor samples}

A total of 426 tumor samples from 256 NF1 patients were studied. NF1 status was based on established clinical consensus criteria [27], with various features abstracted from the clinical records. In addition, a set of 99 MPNST samples (18 non-syndrome associated, 2 schwannomatosisassociated, and 79 of unknown NF1 status) from 81 patients were also studied. All histologic sections were reviewed by a board certified neuropathologist (FJR) and classified to the extent feasible using criteria embodied in the 2016 WHO Classification of Tumors of the Central Nervous System [23] and recently proposed criteria for nerve sheath tumors $[26,30]$. Tumor samples included whole sections $(N=364)$ or sections obtained from 3 different TMAs $(N=161)$ consisting of NF1-associated gliomas and MPNSTs that have been previously characterized [24, 32, 36]. All ethical standards were followed and the study was performed under IRB approval, with consent or waiver of consent approval.

\section{Telomere-specific FISH and CISH}

ALT status was performed and interpreted using previously published criteria and was characterized by the presence of distinct large telomeric DNA signals using fluorescence in situ hybridization (FISH) [12, 13, 39] or chromogenic in situ hybridization (CISH) [39] assays. In brief, tissue slides were deparaffinized, hydrated, and steamed for $25 \mathrm{~min}$ in citrate buffer (Vector Laboratories). This was followed by dehydration and hybridization with a
Cy3-labeled peptide nucleic acid (PNA) probe complementary to the mammalian telomere repeat sequence [(N-terminus to C-terminus) CCCTAACCCTAACCCT AA]. In the FISH approach, an Alexa Fluor-488-labeled PNA probe specific to human centromeric DNA repeats was also included as a control to assess the validity of the hybridization. Following post-hybridization washes, the slides were counterstained with DAPI following posthybridization washes. The telomere-specific CISH was previously outlined. Briefly, deparaffinized slides were hydrated, steamed for $25 \mathrm{~min}$ in citrate buffer, dehydrated, and hybridized with a Cy3-labeled PNA probe (see above). Sections were blocked against endogenous peroxidase activity with Dual Endogenous Enzyme-Blocking Agent (Dako) for $10 \mathrm{~min}$, incubated with a monoclonal anti-Cy3/ Cy5 antibody (ab52060, Abcam, 1:2500) for $1 \mathrm{~h}$ at room temperature, then incubated with an anti-mouse secondary antibody (Leica Microsystems) for $30 \mathrm{~min}$, and detected with 3,30-diaminobenzidine (Sigma-Aldrich) after $10 \mathrm{~min}$. Finally, sections were counterstained with hematoxylin, rehydrated, and mounted for visual inspection. In the subset of ALT-negative cases with appropriate hybridization signals in the internal non-neoplastic components, telomere lengths were qualitatively scored by direct visual assessment of stained slides comparing telomere signals from tumor cells with telomere signals from benign cells (entrapped non-neoplastic cells) from the same case. In all cases, signals from benign cells were considered $2+$ (normal). Telomere signals in tumor cells of different cases ranged from short (1+) to normal (2+) to long (3+).

\section{Immunohistochemistry}

Immunohistochemical studies that were previously performed as part of the clinical diagnostic workup were reviewed. However, in most cases immunohistochemical studies were systematically performed using antibodies recognizing either ATRX, DAXX, or H3K27me3.

Immunohistochemical studies were systematically performed in most cases using an ATRX antibody (Rabbit polyclonal, 1:200 dilution, catalog\# HPA001906 SigmaAldrich). Immunostaining was performed on automated instruments (BenchMark, Ventana Medical Systems, Tucson, AZ, USA). The immunohistochemical protocol included deparaffinization, hydration, antigen retrieval, primary antibody incubation, and detection and visualization as per manufacturer's instructions. Immunohistochemistry for DAXX (Rabbit polyclonal, 1:100 dilution, catalog\# HPA008736, Atlas Antibodies) was performed manually. Sections were incubated with primary antibody for $2 \mathrm{~h}$ at room temperature followed by secondary antibody (Leica Microsystems) for $30 \mathrm{~min}$ and detected with 3,30-diaminobenzidine (Sigma-Aldrich) after $10 \mathrm{~min}$. Immunohistochemistry for H3K27me3 was performed manually in whole tissue sections using a mouse monoclonal antibody 
(Abcam, Cat\# ab6002, Cambridge, MA) at 1:1600 dilution an incubated overnight at $4{ }^{\circ} \mathrm{C}$. The subset of MPNST cases studied in a TMA were tested for H3K27me3 status as part of a previous study [32]. Immunoreactivity preservation in internal non-neoplastic cell components was required for valid interpretation in all cases to evaluate ATRX, DAXX, or H3K27me3 loss. In cases where the loss was clearly limited to a subset of neoplastic cells, with preserved reactivity in non-neoplastic components, the loss was interpreted as "partial".

\section{Next generation sequencing}

To identify possible somatic genetic alterations associated with ALT, we performed next generation sequencing on the 9 ALT-positive tumors with available tissue (6 gliomas and 3 MPNST). Adjacent formalin-fixed, paraffin-embedded sections cut at $5-\mu \mathrm{m}$ thick were scrapped into $2 \mathrm{ml}$ tubes for DNA extraction, focusing on areas enriched for neoplastic cells. An automated Siemens Tissue Preparation System (Siemens Healthcare Diagnostics, Inc., Tarrytown, NY) was used for DNA extraction and purification. Quantification of Genomic DNA was performed using the Qubit 2.0 Fluorometer (Life Technologies, Carlsbad, CA). DNA libraries were prepared using Agilent SureSelect-XT reagents (Agilent Technologies, Inc., Santa Clara, CA). Genomic regions of interest were captured using an Agilent customdesigned bait set covering the full coding regions of 644 cancer associated genes, although exon 1 is poorly covered for some genes. Genes of interest included NF1, SUZ12, EED, ATRX, DAXX, and BRAF. Sequencing of libraries was achieved to an average unique read depth of greater than 500X using Sequencing by Synthesis (SBS) $2 \times 100$ base pairs $(\mathrm{bp})$ paired-end cluster generation on the Illumina HiSeq 2500 plataform (Illumina, Inc., San Diego, CA). FASTQ files were generated from Binary Cluster Files (.bcl) using the Illumina bcl2fastq v1.8.4 software with parameters set as per vendor's specifications. FASTQ files were aligned to the human genome reference hg19 (GRCh37) using the Burrows-Wheeler Aligner v0.7.10 algorithm with default settings. BAM files (.bam) were generated using Picard Tools v1.119 and variant calling was performed using in-house variant caller algorithm (MDLVC v5.0) cross referenced with HaplotypeCaller (Genome Analysis Tool Kit 3.3) under discovery mode in the coding regions of target genes. All variant calls were inspected using Integrated Genomics Viewer v2.3.4 (IGV; Broad Institute, MIT Harvard, Cambridge, MA) and annotated with dbSNP v150 and COSMIC v82 databases. Common single nucleotide polymorphisms (SNPs; population allele frequency $>50 \%$ ) were excluded from analysis. Matched normal (tissue or peripheral blood) was not available for comparison. Mean target coverage of the full coding regions (including exon 1) of $A T R X, D A X X$, and NF1 was 688 reads (330 to 930 average base read depth), 811 reads (406 to 1129 average base read depth), and 823 reads (423 to 1089 average base read depth), respectively, across all cases.

\section{Statistical analysis}

All characteristics were described using proportions, ranges, means, medians, and standard deviations as appropriate. Proportions were compared using Chi-Square or Fisher's exact tests as appropriate. Survival rates were visualized using Kaplan-Meier curves and separations of curves were assessed using the log-rank test. Overall survival was calculated from the time of first pathologic diagnosis to death. Recurrence-free survival was calculated from the time of first pathologic diagnosis to the first evidence of tumor growth per clinical record, or time of death. In further analyses in the glioma and MPNST cohorts, Cox-proportional hazard models were used to assess the independent effects of either telomere length or ALT on overall survival while adjusting for age and grade. Statistical analyses were performed using GraphPad Prism version 8.0 (San Diego, CA), SAS version 9.4 (Cary, NC), and $\mathrm{R}$ version 3.3.2.

\section{Results \\ ALT and long telomeres are frequent in NF1-associated CNS tumors}

A total of 78 CNS tumors from 70 patients with NF1 were analyzed, including 70 primary biopsies or resections and 8 surgeries/recurrences. Clinicopathologic and molecular features are outlined for all CNS tumors (Additional file 1: Table S1) and summarized for the gliomas in Table 1. In the glioma cohort, ALT was present in 23 (of 70; 32.9\%) tumors. When comparing groups, ALT was present in the majority of high-grade gliomas 14 (of 23; 60\%) compared to 9 (of 47; 19\%) low-grade gliomas, a difference that was statistically significant $\left(p=0.0009, \chi^{2}\right.$ test). When focusing on different pathologic subtypes, ALT was present in only 2 (of 26, 8\%) NF1-associated PA compared to 21 (of 44, 47\%) other NF1-CNS (non-PA) tumors $(p=0.001$, Fisher Exact Test). When assessing additional tumor samples/recurrences, there was a perfect concordance among multiple resections from the same patient, with ALT present in 3 and absent in 5 pathology samples from the same patient. Among 23 cases with ALT, ATRX loss was present in $17(74 \%)$ (14 complete, 3 partial) and preserved in 6 (26\%). In contrast, DAXX was preserved in 11 (of 11) cases. Finally, among the ALT-negative cases, 3 (of 42; 7\%) cases tested demonstrated partial ATRX loss, while DAXX was preserved in 14 (of 14) cases.

Next, telomere lengths were evaluated in the ALTnegative tumors, which was possible in 30 cases. Representative images of telomere lengths in NF1-associated 
Table 1 Clinico-pathologic and molecular features of gliomas, MPNST, and neurofibromas

\begin{tabular}{llll}
\hline & $\begin{array}{l}\text { Gliomas } \\
(N=70)\end{array}$ & $\begin{array}{l}\text { MPNST } \\
(N=152)\end{array}$ & $\begin{array}{l}\text { Neurofibromas } \\
(N=77)^{\mathrm{a}}\end{array}$ \\
\hline Mean age (SD) & $20.6(13.8)$ & $38.9(17.3)$ & $30.6(17.7)$ \\
Gender (\%) & & & \\
Male & 52.9 & 49.0 & 48.1 \\
Female & 47.1 & 51.0 & 52.0 \\
Grade (\%) & & & \\
High & 32.9 & 88.7 & - \\
Low & 67.1 & 11.3 & - \\
ALT status (\%) & & & - \\
Positive & 32.9 & 16.6 & - \\
Negative & 67.1 & 81.5 & - \\
Missing & - & 2.0 & - \\
Telomeres (\%) & & & - \\
Normal & 24.3 & 9.9 & - \\
Short & 1.4 & 9.3 & - \\
Long & 17.1 & 0.7 & - \\
ALT & 32.9 & 16.6 & - \\
Missing & 24.3 & 63.6 & - \\
NF status (\%) & & 61.6 & - \\
NF1 & - & - & \\
Unknown & - & & - \\
\hline
\end{tabular}

${ }^{a}$ Multiple samples tested per patient (77 patients with a mean of 2.4 samples per individual)

gliomas are illustrated (Fig. 1a-c). Telomere lengths were normal in 17 (57\%) cases and abnormally long in 12 (40\%). Thus, ALT was a feature of predominantly highgrade or non-PA NF1-associated gliomas, while abnormally long telomeres (in the absence of ALT) are present in a subset of low-grade astrocytomas.

\section{ALT and short telomere lengths are limited to malignant subtypes among NF1-associated nerve sheath tumors}

Next, we studied 197 samples obtained from 174 MPNSTs from 152 patients (93 NF1-associated, 16 non-syndromeassociated, 1 Schwannomatosis-associated, and 42 with unknown syndrome status given incomplete clinical history). Clinicopathologic and molecular features are outlined for all nerve sheath tumors (Additional file 1: Table S1) and summarized for the MPNST and neurofibroma cohorts in Table 1. ALT was present in 25 (of 148; 17\%) MPNSTs from unique patients. In contrast, ALT was present in only 2 (of 193) neurofibromas obtained from 77 NF1 patients, a statistically significant difference $(p<0.0001$, Fisher Exact Test). Notably, the 2 ALT-positive neurofibromas were obtained from a single patient (case 78) who underwent malignant transformation into an ALTpositive MPNST (Additional file 1: Figure S1). Tumors from this patient demonstrated partial ATRX and DAXX protein loss but lacked definite $A T R X$ and $D A X X$ mutations (Additional file 1: Table S1 and Table S2).

We assessed telomere lengths in a subset of ALTnegative MPNST cases $(n=30)$, identifying $14(47 \%)$ cases with short, 15 (50\%) cases with normal, and 1 (3\%) case with long telomere lengths. Representative images of telomere lengths in MPNST are illustrated in Fig. 1df. When limiting to only the NF1-associated group, ALT was present in 9 (12\%), while telomere lengths were abnormally short in 9 (of 24 ALT-negative cases; 38\%), normal in 14 (58\%), and long in a single case (4\%). When limiting to only the sporadic group, ALT was present in 2 (of 14; 14\%) MPNSTs, and telomere lengths were all short in the 5 evaluable ALT-negative cases. A total of 183 (of 191) ALT-negative neurofibromas were evaluable for telomere lengths and all cases displayed normal telomere lengths.

Within the ALT-positive MPNSTs, 6 (of 10) from distinct patients had ATRX protein loss, which was complete in 2 and partial in 4. Partial DAXX loss was present in 1 (of 10) cases. In this group, all 10 cases showed retained H3K27 trimethylation. In addition, one MPNST case displayed ATRX protein loss and was ALT-positive in the primary, and also displayed ATRX protein loss and was ALT-positive in 3 additional distant metastases, suggesting that ATRX loss and ALT are maintained during tumor progression. When assessing the ALT-negative cases, 11 (of 53; 21\%) had partial ATRX loss and 3 (of 32; 9\%) had partial DAXX loss. These findings collectively suggest that abnormal telomere lengths are frequent in MPNST, in contrast to their benign neurofibroma counterparts, and are characterized by the presence of ALT or abnormally shortened telomeres.

Next, we evaluated other rare NF1-associated solid tumors $(n=46)$. A single malignant phyllodes tumor of the breast demonstrated ALT; however, retained ATRX protein expression. In contrast, none of the other rare NF1associated tumors demonstrated ALT (Additional file 1: Table S3), including gastrointestinal stromal tumors (GIST), pheochromocytomas, glomus tumors, juvenile xanthogranulomas, and duodenal neuroendocrine tumors.

\section{Genetic alterations associated with ALT in NF1-associated tumors}

To study genetic alterations associated with the development of ALT, we performed NGS in 4 MPNSTs (3 ALT-positive and 1 ALT-negative) and 6 ALT-positive gliomas, 4 of which were also analyzed as part of a prior study [31]. Sequencing results of ALT-positive cases are provided by case in Additional file 1: Table S2. In the ALT-positive glioma group, all 6 had pathogenic NF1 mutations, with a high VAF suggestive of LOH $(n=4)$ or a second mutation $(n=1)$. Three (of 6) had ATRX 


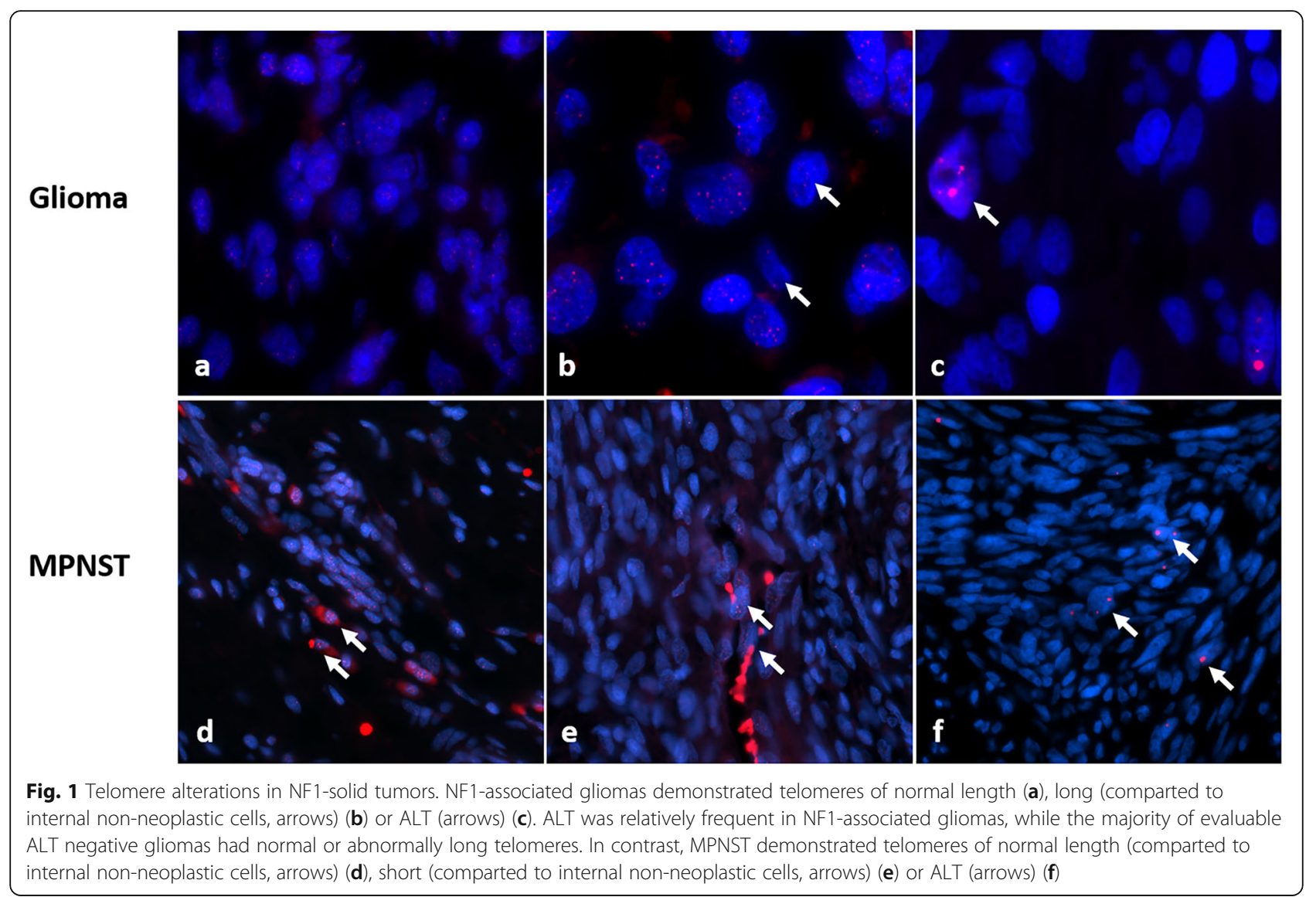

mutations, 2 had homozygous $C D K N 2 A / B$ deletions, but none had a $D A X X$ mutation.

All 3 ALT-positive MPNST had pathogenic NF1 mutations and lacked EED, SUZ12, or DAXX mutations. Case 110 had an $A T R X$ p.E511Kfs*3 mutation with ATRX protein loss (Fig. 2). Case 78 had an ATRX variant (p.Q929E) with a 67\% VAF and partial ATRX protein loss; whereas case 53 lacked an ATRX mutation, but contained two RECQL4 variants (Additional file 1: Table S2). RECQL4 is a DNA helicase and alterations in genes associated with DNA repair could lead to ALT, in the absence of the better known alterations in the chromatin remodelers ATRX and DAXX. RECQL4 variants were also present in two ALT-positive, ATRX intact NF1-gliomas. The ALTnegative MPNST with sequencing data displayed NF1 and SUZ12 mutations. Next, we analyzed publicly available gene sequencing data from cBioPortal $[6,11]$ and identified missense DAXX mutations in 2 (of 15) MPNSTs, both NF1-associated. These tumors lacked PRC2 (EED, SUZ12) mutations, and no mutations were present in $R B L 2$ or $S P 100$, two altered telomere maintenance genes found to be enriched in ALT-positive leiomyosarcomas [7]. Collectively, these findings suggest that in a subset of NF1associated tumors, ALT may be mediated by genes other than $A T R X$ and $D A X X$.
ALT is associated with shorter overall survival in NF1associated glioma

In the NF1-glioma cohort, median overall survival for patients with an ALT-positive tumor was only 18 months and significantly differed compared to patients with either long (69 months) or normal telomeres (not reached) $(p<0.0001, \log$ rank test). When restricting the analysis to cases with evaluable telomere lengths, there was also a trend for intermediate survival for patients with long telomeres and better survival for patients with normal telomeres, although the differences in survival appeared to be driven mostly by the ALT-positive group $(p=0.0021, \log$ rank test) (Fig. 3a, b). When modeling in a Cox univariate model, we observed consistent patterns for altered telomeres lengths (Long: $H R=3.99$, 95\% CI $=0.70-22.77$; ALT HR $=13.05,95 \% \mathrm{CI}=2.35$ 72.43) when compared to normal telomere lengths (Additional file 1: Table S4).

Since age and grade are some of the most important prognostic factors for central nervous system tumors, and ALT was associated with adults and higher grade tumors in our prior study [39], we performed multivariate analyses adjusting for these two factors. While there was not a statistically significant difference by telomere length group (Additional file 1: Table S4), ALT status 

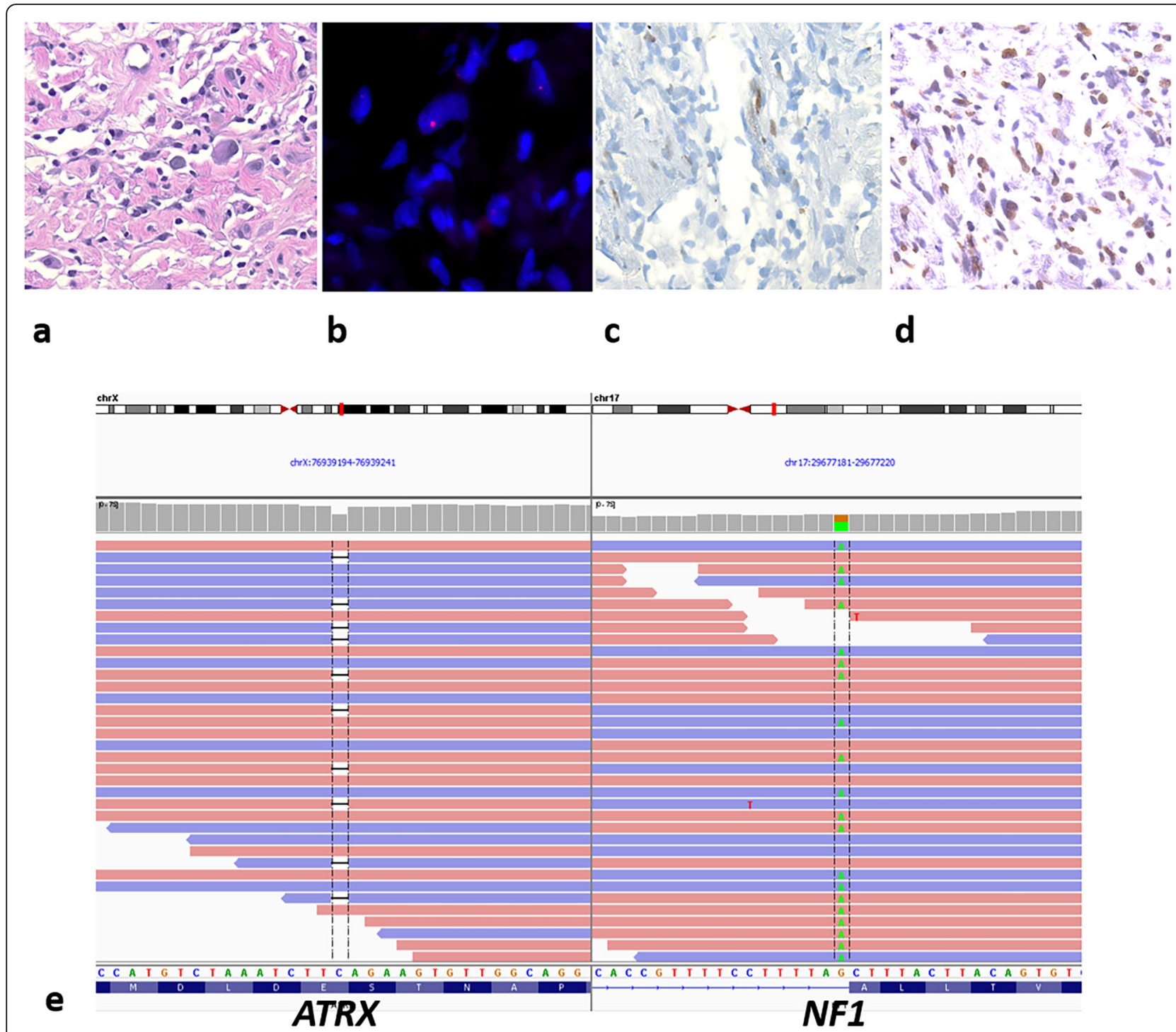

Fig. 2 ALT-positive MPNST represent a distinct molecular subgroup. ALT-positive NF1-MPNST (case 114) (a), with ALT (b), ATRX protein loss (c), but preserved H3K27 trimethylation (d). Next generation sequencing demonstrated concurrent ATRXp.E511Kfs*3 and a NF1 splice site mutation (c.7259-1G > A) (e), but no EED or SUZ12 mutations

remained significantly associated with a worse outcome in the model $(\mathrm{HR}=3.62$, 95\% CI $1.17-11.17 ; p=0.03)$ (Table 2).

\section{Shorter telomeres are associated with better overall survival in NF1-associated MPNST}

In the NF1-MPNST cohort, overall survival was decreased for patients with ALT-positive tumors, intermediate for those with normal telomeres, and increased for those with short telomeres $(p=0.0052)$. When evaluating the entire MPNST cohort (including sporadic tumors and tumors with unknown NF1-status), median survival for patients was 28 months for ALT-positive tumors, 31 months for ALT-negative tumors, 32 months for normal telomeres, and not reached for short telomeres (Additional file 1:
Figure S2a,b). These survival differences were similar in the NF1-MPNST group ( $p=0.0016)$ (Fig. 3c,d).

In contrast to the glioma cohort, there were no statistical significant differences in overall survival between ALT-positive and ALT-negative NF1-MPNSTs $(p=0.20)$. Similarly, using a Cox univariate model, the altered telomere length groups did not differ (Short HR $=0.13$, 95\% $\mathrm{CI}=0.02-1.12$; ALT HR $=1.93,95 \% \mathrm{CI}=0.68-5.49)$ when compared to the normal telomere group. However, in a multivariate model with age and grade, the short telomere group displayed a significantly better overall survival difference $(\mathrm{HR}=0.03,95 \% \mathrm{CI}=0.01-0.29 ; p=0.003)$ compared to the normal telomere group (Table 3). When considering only ALT status, there were no significant differences with ALT-positivity in the NF1-MPNST cohort 


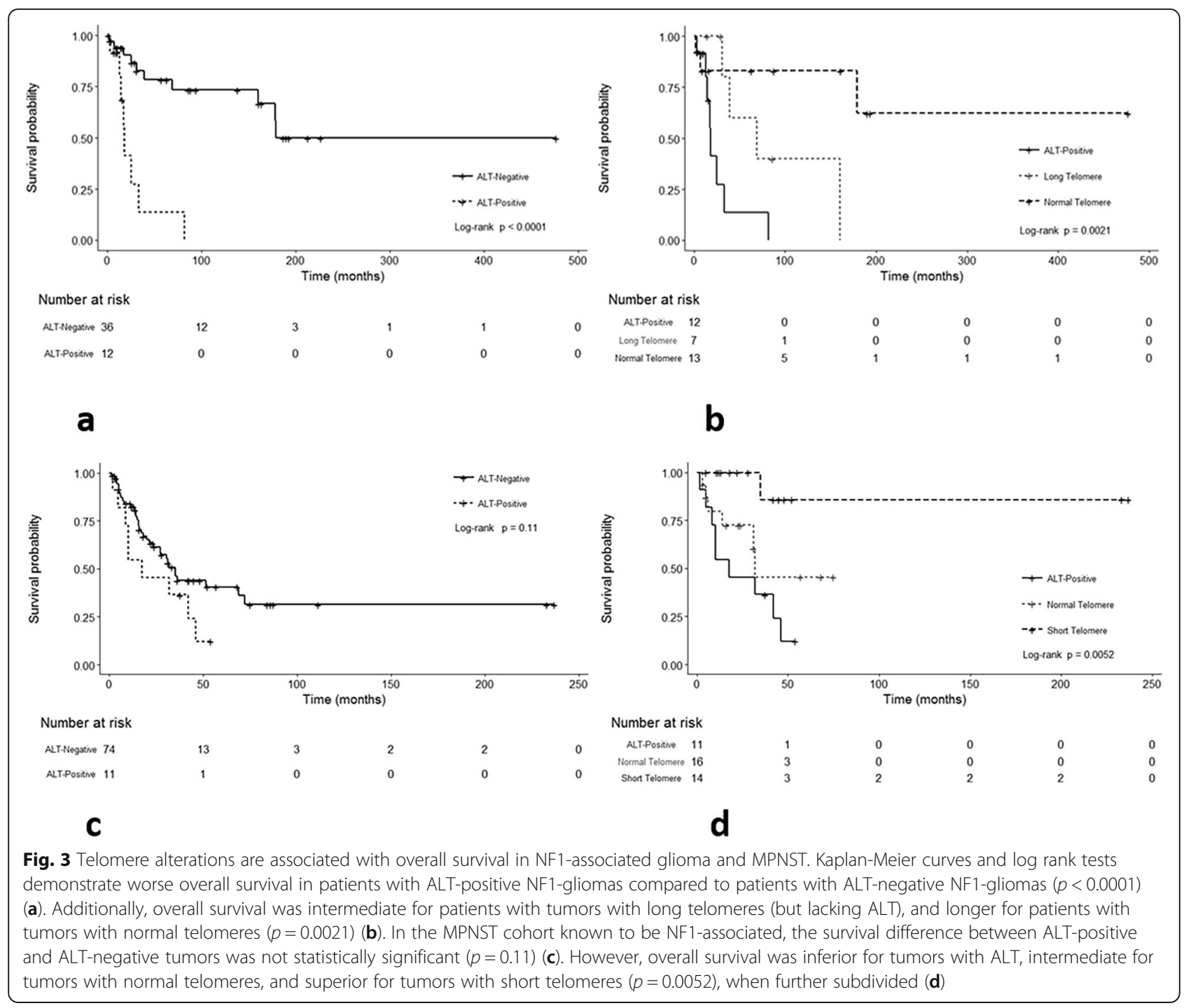

Table 2 Association of ALT status with overall survival in patients with glioma

\begin{tabular}{|c|c|c|c|c|c|c|c|c|}
\hline & \multirow[b]{2}{*}{ Death/Censor } & \multirow[b]{2}{*}{ Person-time (months) } & \multicolumn{3}{|c|}{ Univariate } & \multicolumn{3}{|c|}{ Multivariate } \\
\hline & & & $\mathrm{HR}$ & $(95 \%$ Cl) & $P$-value & $\mathrm{HR}$ & $(95 \% \mathrm{Cl})$ & P-value \\
\hline Age & $18 / 30$ & 3469 & 1.05 & $(1.02-1.08)$ & 0.001 & 1.02 & $(0.98-1.05)$ & 0.3 \\
\hline \multicolumn{9}{|l|}{ Grade } \\
\hline Low & $6 / 25$ & 2762 & 1.00 & (Ref) & - & 1.00 & (Ref) & - \\
\hline High & $12 / 5$ & 707 & 6.69 & $(2.30-19.41)$ & 0.0005 & 3.36 & $(0.94-12.10)$ & 0.06 \\
\hline \multicolumn{9}{|l|}{ ALT status } \\
\hline Negative & $10 / 26$ & 3221 & 1.00 & (Ref) & - & 1.00 & (Ref) & - \\
\hline Positive & $8 / 4$ & 248 & 7.86 & $(2.68-23.06)$ & 0.0002 & 3.62 & $(1.17-11.17)$ & 0.03 \\
\hline
\end{tabular}


Table 3 Association of telomere lengths with overall survival in patients with NF1-associated MPNST

\begin{tabular}{|c|c|c|c|c|c|c|c|c|}
\hline & \multirow[b]{2}{*}{ Death/Censor } & \multirow[b]{2}{*}{ Person-time (months) } & \multicolumn{3}{|c|}{ Univariate } & \multicolumn{3}{|c|}{ Multivariate } \\
\hline & & & $\mathrm{HR}$ & $(95 \% \mathrm{Cl})$ & P-value & $\mathrm{HR}$ & $(95 \% \mathrm{Cl})$ & P-value \\
\hline Age & $16 / 25$ & 1482 & 1.01 & $(0.98-1.04)$ & 0.5 & 1.08 & $(1.02-1.14)$ & 0.006 \\
\hline \multicolumn{9}{|l|}{ Grade } \\
\hline Low & $1 / 3$ & 168 & 1.00 & (Ref) & - & 1.00 & (Ref) & - \\
\hline High & $15 / 22$ & 1315 & 2.29 & $(0.30-17.52)$ & 0.3 & 12.33 & $(0.42-28.97)$ & 0.03 \\
\hline \multicolumn{9}{|c|}{ Telomere lengths } \\
\hline Normal & $6 / 10$ & 417 & 1.00 & (Ref) & - & 1.00 & (Ref) & - \\
\hline Short & $1 / 13$ & 801 & 0.13 & $(0.02-1.12)$ & 0.06 & 0.03 & $(0.01-0.29)$ & 0.003 \\
\hline ALT & $9 / 2$ & 264 & 1.93 & $(0.68-5.49)$ & 0.2 & 1.21 & $(0.41-3.59)$ & 0.7 \\
\hline
\end{tabular}

$(\mathrm{HR}=1.21,95 \% \mathrm{CI}=0.41-3.59 ; p=0.70)($ Table 3$)$ or in the entire MPNST group (including sporadic and unknown status) (Additional file 1: Table S5 and Table S6).

\section{Discussion}

Identifying the genetic drivers and the precise sequence of genetic hits in NF1-associated tumor development is still being fully elucidated. However, we have previously demonstrated that functional loss of ATRX and activation of ALT are frequent features of diffuse and highgrade astrocytomas that develop in patients with NF1, and are present in 7 (58\%) and 8 (67\%) respectively [39]. More recently, D'angelo et al. identified ATRX mutations in $38 \%$ of NF1-associated high grade gliomas, compared to $3.1 \%$ of low-grade gliomas [8]. In addition, another group of aggressive gliomas characterized by a high frequency of ATRX alterations and ALT activation is PA with anaplasia, approximately one third of which are NF1-associated or demonstrate somatic NF1 gene mutations [35, 37]. One important finding in our current study is that in NF1-associated gliomas, ALT is independently associated with worse survival in a multivariate model also accounting for grade and age, two key factors consistently associated with survival in gliomas.

ALT was also identified in one additional tumor type in our study (i.e. MPNST) albeit at a lower frequency (17\%) compared to the gliomas. Only one patient had two ALT-positive neurofibromas in our study, and they were presumably precursors to a fatal ALT-positive MPNST. ALT was also identified in a single malignant phyllodes tumor, although ALT was uniformly absent in the remaining NF1-associated tumors (e.g. GIST, neuroendocrine tumors, and glomus tumors), suggesting that ALT in NF1-associated tumors is associated with a malignant phenotype. Our results are in agreement with the finding of ALT in 3 (of 14;21\%) MPNST in a prior study, only one of which had ATRX loss [22]. In addition, an ATRX variant was found in 1 (of 7) NF1associated MPNSTs through next generation sequencing in a previous study [15]. More recently, Lu et al. reported aberrant ATRX immunoreactivity in 65\% of NF1-associated MPNST, a finding associated with shorter overall survival [24]. Of note, in cBioPortal, $A T R X$ mutations were present in 4 (2.5\%) of 162 pheochromocytomas/ paragangliomas, another tumor type that develops in NF1 patients. Others have reported ATRX mutations in $12.6 \%$ of pheochromocytomas/paragangliomas, mostly associated with $\mathrm{SDH}$ alterations, but also in one tumor with an NF1 mutation [10]. Additionally, telomerase activation and $A T R X$ mutations were found to be independent factors for poor prognosis in pheochromocytomas/paragangliomas in a recent study [17]. Loss of ATRX expression and ALT are very rare in GIST in general, which is in accordance with our findings $[1,22]$. However, we did not identify ATRX alterations in one of the 3 ALT-positive MPNST sequenced. We recently studied a group of low-grade gliomas developing in NF1 patients ("SEGA-like astrocytomas") [16]. We have included these cases as part of this broader study, and found ALT was present in 4 (of 10; 40\%) of these tumors. However, only one of these ALT-positive tumors contained a pathogenic $A T R X$ mutation. These findings suggest that ALT may develop independently of ATRX and DAXX in subsets of NF1-associated tumors.

Another key finding in our study is that short telomeres were prevalent in ALT-negative MPNST, while long telomeres were present in ALT-negative gliomas, independent of grade. Telomere lengths in cancer cells can vary among different tumor types. For example, a systematic analysis of telomere lengths using sequencing data found that in most tumors telomeres were shorter than in normal tissues; however, longer telomeres were observed in gliomas and sarcomas [2]. These differences potentially reflect different age distributions, variation of telomere lengths among cell lineages, or may even reflect differences in the degree and timing of telomerase activation.

Recent studies in MPNST have demonstrated frequent mutations in SUZ12 and EED which encode protein components of the PRC2 complex [21, 43]. These alterations are associated with loss of H3K27me3 [32, 33, 


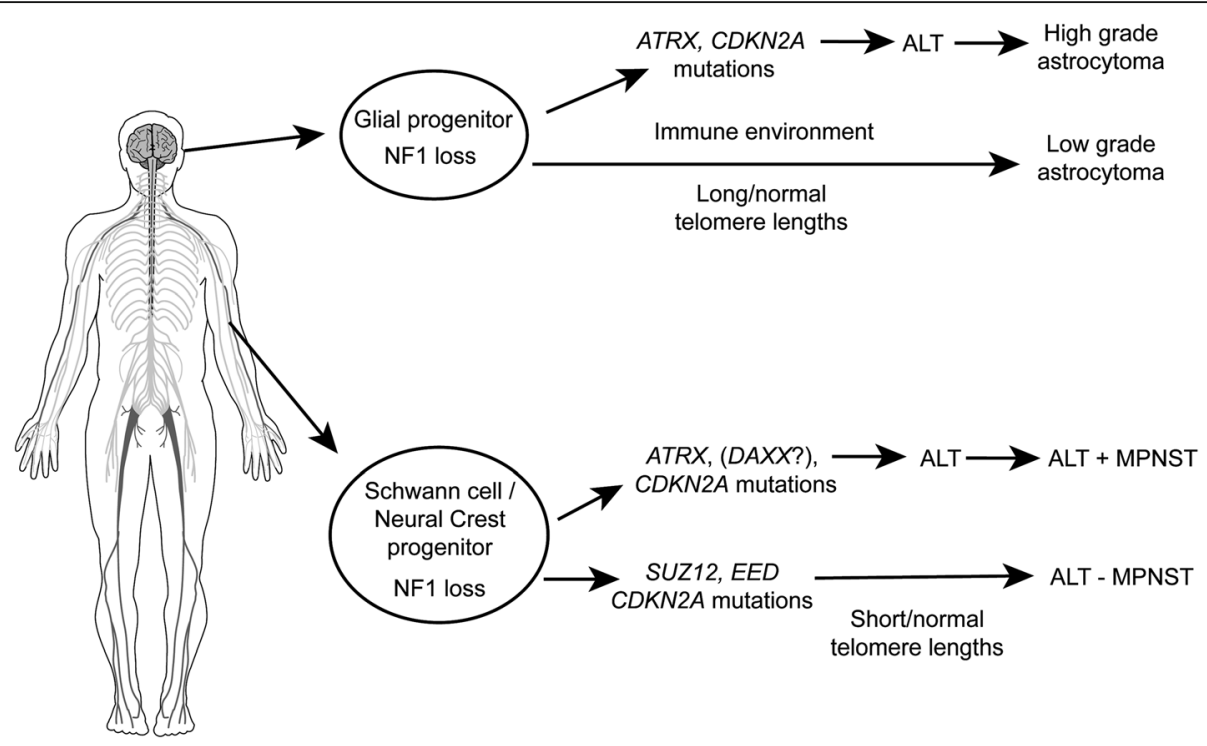

Fig. 4 Telomere alterations and contribution to NF1 tumorigenesis. In gliomagenesis, ATRX alterations and ALT are associated with high grade tumors, while longer telomeres (without ALT) are frequent in both high grade and low grade gliomas. In malignant nerve sheath tumors, there are two separate pathways, one that involves ALT (with or without ATRX alterations), and another that lacks ALT and ATRX alterations, but frequently has SUZ12 or EED alterations, loss of H3 K27 trimethylation and abnormally short telomeres. Some alterations are common to all malignant NF1-associated tumors, particularly CDKN2A deletions/mutations

40]. However, loss of H3K27me3 was not a feature in MPNST with ALT, and we did not identify SUZ12 or EED mutations. These findings suggest that ALT-positive MPNST represents a distinctive molecular subgroup that may benefit from different therapeutic approaches (Fig. 4).

Prior studies suggest that ATRX mutation alone is not sufficient for tumorigenesis or the development of ALT. Rather, ATRX mutations in CNS tumors seem to develop in the context of other more basic genetic drivers to facilitate tumorigenesis. In the context of NF1-associated tumors, this seems to be NF1 loss, while IDH mutations and histone $\mathrm{H} 3$ mutations (particularly G34) frequently coexist with ATRX loss in other tumor subsets [5, 20, 42]. In the rare phenomenon of pilocytic astrocytomas with anaplasia, ATRX loss frequently co-occurs with NF1 loss, as well as mutually exclusive alterations in the members of the MAPK pathway (e.g. BRAF) [35, 37]. Detecting ATRX alterations may also have management implications for NF1 patients with gliomas, since when present they identify subgroups that are clinically more aggressive. ATRX loss is routinely tested for in the laboratory setting through immunohistochemistry or sequencing. In contrast, while ALT is not tested for clinically, it has the potential to be used in cases with equivocal ATRX findings, either as by FISH or CISH.

One of the limitations of our studies includes that we did not find a specific explanation for the development of ALT in a subset of cases lacking ATRX or DAXX mutations. The NGS panel applied covers many genes, but it could have missed other ALT related genes that in the future may be identified by more comprehensive (whole exome/genome) sequencing methods. Additionally, the number of rare NF1-associated solid tumors in our study is relatively low. Although no clear patterns for telomere alterations were noted, future studies with larger number of cases may be required for confirmation of our findings.

In summary, our data support an important role for ATRX/DAXX loss and acquisition of ALT with an aggressive biology in NF1-associated gliomas, as well as a molecular subset of NF1-associated MPNSTs.

\section{Additional file}

Additional file 1: Figure S1. MPNST case 78. Figure S2. Telomere alterations are associated with overall survival in combined MPNST group. Table S1. Clinicopathologic Features of NF1-Associated Solid Tumors and MPNST (337 patients). Table S2. Next generation sequencing results of ALT positive NF1-associated gliomas and MPNST $(n=9)$. Table S3. Telomere alterations in rare NF1-associated tumors $(n=46)$. Table S4. Association of telomere length with overall survival in patients with glioma. Table S5. Association of telomere lengths with overall survival in all patients with MPNST. Table S6. Association of ALT with overall survival in all patients with MPNST. (PDF 3427 kb)

\section{Acknowledgments}

This work was supported in part by Pilocytic/Pilomyxoid Fund, including Lauren's First and Goal, and the Stick it to Brain Tumors Annual Women's Ice Hockey Tournament (FJR), the Basic/Translational Science Investigator Award from the North American Neuroendocrine Tumor Society supported by the Neuroendocrine Tumor Research Foundation $(\mathrm{CMH})$, a collaborative grant between the Wilmer Eye Institute (Baltimore, MD) and the King Khaled Eye Specialist Hospital, Riyadh, Saudi Arabia (to FJR), Department of Defense grant W81XWH-18-1-0496 (FJR), NIH grant 2T32CA009110-39A1 (JAB), and 
$\mathrm{NIH}$ grant P30 CA006973 to the Sidney Kimmel Comprehensive Cancer Center (PI: W. Nelson).

\section{Author details}

${ }^{1}$ Departments of Pathology, Johns Hopkins University School of Medicine, Sheikh Zayed Tower, Room M2101, 1800 Orleans Street, Baltimore, MD 21231, USA. ²Departments of Ophthalmology, Johns Hopkins University School of Medicine, Sheikh Zayed Tower, Room M2101, 1800 Orleans Street, Baltimore, MD 21231, USA. ${ }^{3}$ Sidney Kimmel Comprehensive Cancer Center, Johns Hopkins University School of Medicine, Sheikh Zayed Tower, Room M2101, 1800 Orleans Street, Baltimore, MD 21231, USA. ${ }^{4}$ Departments of Neurology, Johns Hopkins University School of Medicine, Sheikh Zayed Tower, Room M2101, 1800 Orleans Street, Baltimore, MD 21231, USA. ${ }^{5}$ Department of Epidemiology, Johns Hopkins Bloomberg School of Public Health, Baltimore, MD, USA. ${ }^{6}$ Department of Laboratory Medicine and Pathology, Mayo Clinic, Rochester, MN, USA. ${ }^{7}$ Department of Pathology, University of California San Francisco, San Francisco, CA, USA. ${ }^{8}$ Department of Pathology and Immunology, Washington University, St. Louis, MO, USA. ${ }^{9}$ University of Arkansas, Little Rock, AR, USA. ${ }^{10}$ Department of Orthopedics, Johns Hopkins University School of Medicine, Baltimore, MD, USA.

${ }^{11}$ Department of Neurosurgery, Johns Hopkins University School of Medicine, Baltimore, MD, USA.

Received: 11 July 2019 Accepted: 19 August 2019 Published online: 28 August 2019

\section{References}

1. Akaike K, Toda-Ishii M, Suehara Y, Mukaihara K, Kubota D, Mitani K, Takagi T, Kaneko K, Yao T, Saito T (2015) TERT promoter mutations are a rare event in gastrointestinal stromal tumors. Springerplus 4:836. https://doi.org/10.1186/ s40064-015-1606-2

2. Barthel FP, Wei W, Tang M, Martinez-Ledesma E, Hu X, Amin SB, Akdemir KC, Seth S, Song X, Wang Q et al (2017) Systematic analysis of telomere length and somatic alterations in 31 cancer types. Nat Genet 49:349-357. https://doi.org/10.1038/ng.3781

3. Brat DJ, Verhaak RG, Aldape KD, Yung WK, Salama SR, Cooper LA, Rheinbay E, Miller CR, Vitucci M, Morozova O et al (2015) Comprehensive, integrative genomic analysis of diffuse lower-grade gliomas. N Engl J Med 372:24812498. https://doi.org/10.1056/NEJMoa1402121

4. Brems H, Beert E, de Ravel T, Legius E (2009) Mechanisms in the pathogenesis of malignant tumours in neurofibromatosis type 1. Lancet Oncol 10:508-515. https://doi.org/10.1016/\$1470-2045(09)70033-6

5. Cancer Genome Atlas Research N, Brat DJ, Verhaak RG, Aldape KD, Yung WK, Salama SR, Cooper LA, Rheinbay E, Miller CR, Vitucci M et al (2015) Comprehensive, integrative genomic analysis of diffuse lower-grade gliomas. N Engl J Med 372:2481-2498. https://doi.org/10.1056/NEJMoa14 02121

6. Cerami E, Gao J, Dogrusoz U, Gross BE, Sumer SO, Aksoy BA, Jacobsen A, Byrne CJ, Heuer ML, Larsson E et al (2012) The CBio cancer genomics portal: an open platform for exploring multidimensional cancer genomics data. Cancer Discov 2:401-404. https://doi.org/10.1158/2159-8290.CD-12-0095

7. Chudasama P, Mughal SS, Sanders MA, Hubschmann D, Chung I, Deeg KI, Wong SH, Rabe S, Hlevnjak M, Zapatka M et al (2018) Integrative genomic and transcriptomic analysis of leiomyosarcoma. Nat Commun 9:144. https:// doi.org/10.1038/s41467-017-02602-0

8. D'Angelo F, Ceccarelli M, Tala GL, Zhang J, Frattini V, Caruso FP, Lewis G, Alfaro KD, Bauchet $L$ et al (2018) The molecular landscape of glioma in patients with Neurofibromatosis 1. Nat Med: Doi. https://doi.org/10.1038/ s41591-018-0263-8

9. Diplas BH, He X, Brosnan-Cashman JA, Liu H, Chen LH, Wang Z, Moure CJ, Killela PJ, Loriaux DB, Lipp ES et al (2018) The genomic landscape of TERT promoter wildtype-IDH wildtype glioblastoma. Nat Commun 9:2087. https:// doi.org/10.1038/s41467-018-04448-6

10. Fishbein L, Khare S, Wubbenhorst B, DeSloover D, D'Andrea K, Merrill S, Cho NW, Greenberg RA, Else T, Montone K et al (2015) Whole-exome sequencing identifies somatic ATRX mutations in pheochromocytomas and paragangliomas. Nat Commun 6:6140. https://doi.org/10.1038/ncomms7140

11. Gao J, Aksoy BA, Dogrusoz U, Dresdner G, Gross B, Sumer SO, Sun Y Jacobsen A, Sinha R, Larsson E et al (2013) Integrative analysis of complex cancer genomics and clinical profiles using the cBioPortal. Sci Signal 6:pl1. https://doi.org/10.1126/scisignal.2004088
12. Heaphy CM, de Wilde RF, Jiao Y, Klein AP, Edil BH, Shi C, Bettegowda C, Rodriguez FJ, Eberhart CG, Hebbar S et al (2011) Altered telomeres in tumors with ATRX and DAXX mutations. Science 333:425. https://doi.org/1 $0.1126 /$ science. 1207313

13. Heaphy CM, Subhawong AP, Hong SM, Goggins MG, Montgomery EA, Gabrielson E, Netto GJ, Epstein Jl, Lotan TL, Westra WH et al (2011) Prevalence of the alternative lengthening of telomeres telomere maintenance mechanism in human cancer subtypes. Am J Pathol 179: 1608-1615. https://doi.org/10.1016/j.ajpath.2011.06.018

14. Heaphy CM, Yoon GS, Peskoe SB, Joshu CE, Lee TK, Giovannucci E, Mucci LA, Kenfield SA, Stampfer MJ, Hicks JL et al (2013) Prostate cancer cell telomere length variability and stromal cell telomere length as prognostic markers for metastasis and death. Cancer Discov 3:1130-1141. https://doi. org/10.1158/2159-8290.CD-13-0135

15. Hirbe AC, Kaushal M, Sharma MK, Dahiya S, Pekmezci M, Perry A, Gutmann DH (2017) Clinical genomic profiling identifies TYK2 mutation and overexpression in patients with neurofibromatosis type 1-associated malignant peripheral nerve sheath tumors. Cancer 123:1194-1201. https:// doi.org/10.1002/cncr.30455

16. Jentoft M, Giannini C, Cen L, Scheithauer BW, Hoesley B, Sarkaria JN, AbellAleff PC, Rodriguez EF, Li Y, Rodriguez FJ (2010) Phenotypic variations in NF1-associated low grade astrocytomas: possible role for increased mTOR activation in a subset. Int J Clin Exp Pathol 4:43-57

17. Job S, Draskovic I, Burnichon N, Buffet A, Cros J, Lepine C, Venisse A, Robidel E, Verkarre V, Meatchi T et al (2019) Telomerase activation and ATRX mutations are independent risk factors for metastatic Pheochromocytoma and Paraganglioma. Clin Cancer Res 25:760-770. https://doi.org/10.1158/1 078-0432.CCR-18-0139

18. Jones RE, Grimstead JW, Sedani A, Baird D, Upadhyaya M (2017) Telomere erosion in NF1 tumorigenesis. Oncotarget 8:40132-40139. https://doi.org/1 0.18632/oncotarget.16981

19. Killela PJ, Reitman ZJ, Jiao Y, Bettegowda C, Agrawal N, Diaz LA Jr, Friedman AH, Friedman H, Gallia GL, Giovanella BC et al (2013) TERT promoter mutations occur frequently in gliomas and a subset of tumors derived from cells with low rates of self-renewal. Proc Natl Acad Sci U S A 110:6021-6026. https://doi.org/10.1073/pnas.1303607110

20. Korshunov A, Capper D, Reuss D, Schrimpf D, Ryzhova M, Hovestadt V, Sturm D, Meyer J, Jones C, Zheludkova O et al (2016) Histologically distinct neuroepithelial tumors with histone 3 G34 mutation are molecularly similar and comprise a single nosologic entity. Acta Neuropathol 131:137-146. https://doi.org/10.1007/s00401-015-1493-1

21. Lee W, Teckie S, Wiesner T, Ran L, Prieto Granada CN, Lin M, Zhu S, Cao Z, Liang Y, Sboner A et al (2014) PRC2 is recurrently inactivated through EED or SUZ12 loss in malignant peripheral nerve sheath tumors. Nat Genet 46: 1227-1232. https://doi.org/10.1038/ng.3095

22. Liau JY, Lee JC, Tsai JH, Yang CY, Liu TL, Ke ZL, Hsu HH, Jeng YM (2015) Comprehensive screening of alternative lengthening of telomeres phenotype and loss of ATRX expression in sarcomas. Mod Pathol 28:15451554. https://doi.org/10.1038/modpathol.2015.114

23. Louis DN, Ohgaki H, Wiestler OD, Cavenee WK, Ellison DW, Figarella-Branger D, Perry A, Reifenberger G, von Deimling A (2016) WHO classification of Tumours of the central nervous system. Revised 4th edition edn. International Agency for Research on Cancer, City

24. Lu HC, Eulo V, Apicelli AJ, Pekmezci M, Tao Y, Luo J, Hirbe AC, Dahiya S (2018) Aberrant ATRX protein expression is associated with poor overall survival in NF1-MPNST. Oncotarget 9:23018-23028. https://doi.org/10.18632/ oncotarget.25195

25. Mantripragada KK, Caley M, Stephens P, Jones CJ, Kluwe L, Guha A, Mautner V, Upadhyaya M (2008) Telomerase activity is a biomarker for high grade malignant peripheral nerve sheath tumors in neurofibromatosis type 1 individuals. Genes Chromosomes Cancer 47:238-246. https://doi.org/10.1002/gcc.20525

26. Miettinen MM, Antonescu CR, Fletcher CDM, Kim A, Lazar AJ, Quezado MM, Reilly KM, Stemmer-Rachamimov A, Stewart DR, Viskochil D et al (2017) Histopathologic evaluation of atypical neurofibromatous tumors and their transformation into malignant peripheral nerve sheath tumor in patients with neurofibromatosis 1-a consensus overview. Hum Pathol 67:1-10. https://doi.org/10.1016/j.humpath.2017.05.010

27. National Institutes of Health Consensus Development Conference Statement (1988) Neurofibromatosis. Arch Neurol 45:575-578

28. Nguyen DN, Heaphy CM, de Wilde RF, Orr BA, Odia Y, Eberhart CG, Meeker AK, Rodriguez FJ (2013) Molecular and morphologic correlates of the 
alternative lengthening of telomeres phenotype in high-grade astrocytomas. Brain Pathol 23:237-243. https://doi.org/10.1111/j.1750-3639.2 012.00630.x

29. Nix JS, Blakeley J, Rodriguez FJ (2019) An update on the central nervous system manifestations of Neurofibromatosis type 1. Acta Neuropathol

30. Ortonne N, Wolkenstein P, Blakeley JO, Korf B, Plotkin SR, Riccardi VM, Miller DC, Huson S, Peltonen J, Rosenberg A et al (2018) Cutaneous neurofibromas: current clinical and pathologic issues. Neurology 91:S5-S13. https://doi.org/10.1212/WNL.0000000000005792

31. Palsgrove DN, Brosnan-Cashman JA, Giannini C, Raghunathan A, Jentoft M, Bettegowda C, Gokden M, Lin D, Yuan M, Lin MT et al (2018) Subependymal giant cell astrocytoma-like astrocytoma: a neoplasm with a distinct phenotype and frequent neurofibromatosis type-1-association. Mod Pathol 31:1787-1800. https://doi.org/10.1038/s41379-018-0103-x

32. Pekmezci M, Cuevas-Ocampo AK, Perry A, Horvai AE (2017) Significance of H3K27me3 loss in the diagnosis of malignant peripheral nerve sheath tumors. Mod Pathol 30:1710-1719. https://doi.org/10.1038/modpathol.2017.97

33. Prieto-Granada CN, Wiesner T, Messina JL, Jungbluth AA, Chi P, Antonescu CR (2016) Loss of H3K27me3 expression is a highly sensitive marker for sporadic and radiation-induced MPNST. Am J Surg Pathol 40:479-489. https://doi.org/10.1097/PAS.0000000000000564

34. Ramamoorthy M, Smith S (2015) Loss of ATRX suppresses resolution of telomere cohesion to control recombination in ALT Cancer cells. Cancer Cell 28:357-369. https://doi.org/10.1016/j.ccell.2015.08.003

35. Reinhardt A, Stichel D, Schrimpf D, Sahm F, Korshunov A, Reuss DE, Koelsche C, Huang K, Wefers AK, Hovestadt V et al (2018) Anaplastic astrocytoma with piloid features, a novel molecular class of IDH wildtype glioma with recurrent MAPK pathway, CDKN2A/B and ATRX alterations. Acta Neuropathol 136:273-291. https://doi.org/10.1007/s00401-018-1837-8

36. Rodriguez EF, Scheithauer BW, Giannini C, Rynearson A, Cen L, Hoesley B, Gilmer-Flynn H, Sarkaria JN, Jenkins S, Long J et al (2011) PI3K/AKT pathway alterations are associated with clinically aggressive and histologically anaplastic subsets of pilocytic astrocytoma. Acta Neuropathol 121:407-420 https://doi.org/10.1007/s00401-010-0784-9

37. Rodriguez FJ, Brosnan-Cashman JA, Allen SJ, Vizcaino MA, Giannini C, Camelo-Piragua S, Webb M, Matsushita M, Wadhwani N, Tabbarah A et al (2018) Alternative lengthening of telomeres, ATRX loss and H3-K27M mutations in histologically defined pilocytic astrocytoma with anaplasia. Brain Pathol: Doi. https://doi.org/10.1111/bpa.12646

38. Rodriguez FJ, Perry A, Gutmann DH, O'Neill BP, Leonard J, Bryant S, Giannini C (2008) Gliomas in neurofibromatosis type 1: a clinicopathologic study of 100 patients. J Neuropathol Exp Neurol 67:240-249. https://doi.org/10.1097/ NEN.0b013e318165eb75

39. Rodriguez FJ, Vizcaino MA, Blakeley J, Heaphy CM (2016) Frequent alternative lengthening of telomeres and ATRX loss in adult NF1-associated diffuse and high-grade astrocytomas. Acta Neuropathol 132:761-763. https://doi.org/10.1007/s00401-016-1619-0

40. Schaefer IM, Fletcher CD, Hornick JL (2016) Loss of H3K27 trimethylation distinguishes malignant peripheral nerve sheath tumors from histologic mimics. Mod Pathol 29:4-13. https://doi.org/10.1038/modpathol.2015.134

41. Simpson K, Jones RE, Grimstead JW, Hills R, Pepper C, Baird DM (2015) Telomere fusion threshold identifies a poor prognostic subset of breast cancer patients. Mol Oncol 9:1186-1193. https://doi.org/10.1016/j.molonc.2015.02.003

42. Sturm D, Witt H, Hovestadt V, Khuong-Quang DA, Jones DT, Konermann C, Pfaff E, Tonjes M, Sill M, Bender S et al (2012) Hotspot mutations in H3F3A and IDH1 define distinct epigenetic and biological subgroups of glioblastoma. Cancer Cell 22:425-437. https://doi.org/10.1016/..ccr.2012.08.024

43. Zhang $M$, Wang $Y$, Jones $S$, Sausen M, McMahon $K$, Sharma R, Wang $Q$, Belzberg AJ, Chaichana K, Gallia GL et al (2014) Somatic mutations of SUZ12 in malignant peripheral nerve sheath tumors. Nat Genet 46:1170-1172. https://doi.org/10.1038/ng.3116

\section{Publisher's Note}

Springer Nature remains neutral with regard to jurisdictional claims in published maps and institutional affiliations.

Ready to submit your research? Choose BMC and benefit from:

- fast, convenient online submission

- thorough peer review by experienced researchers in your field

- rapid publication on acceptance

- support for research data, including large and complex data types

- gold Open Access which fosters wider collaboration and increased citations

- maximum visibility for your research: over $100 \mathrm{M}$ website views per year

At BMC, research is always in progress.

Learn more biomedcentral.com/submissions 